\title{
Study design and patient recruitment for the Japan Polyp Study
}

This article was published in the following Dove Press journal:

Open Access Journal of Clinical Trials

21 May 2014

Number of times this article has been viewed

Yasushi Sano ${ }^{1,2}$

Takahiro Fujii ${ }^{3,4}$

Takahisa Matsuda ${ }^{3}$

Yasushi Oda ${ }^{5,6}$

Shin'ei Kudo ${ }^{7}$

Masahiro Igarashi ${ }^{8}$

Hiroyasu lishi9

Kazuhiro Kaneko 1,10

Kinichi Hotta ${ }^{3,11,12}$

Nozomu Kobayashi, ${ }^{3,13}$

Yuichiro Yamaguchi' ${ }^{12}$

Kiyonori Kobayashi ${ }^{8}$

Hideki Ishikawa ${ }^{14}$

Yoshitaka Murakami ${ }^{15}$

Tadakazu Shimoda ${ }^{16}$

Takahiro Fujimori ${ }^{17}$

Yoichi Ajioka ${ }^{18}$

Hirokazu Taniguchi'16

Hiroaki Ikematsu',3

Kazuo Konishi ${ }^{10}$

Yutaka Saito ${ }^{3}$

Shigeaki Yoshida ${ }^{1,19}$

'Department of Gastroenterology, Endoscopy Division, National Cancer Center Hospital East, Kashiwa,

${ }^{2}$ Gastrointestinal Center and Institute of Minimally Invasive Endoscopic Care (iMEC), Sano Hospital, Kobe, ${ }^{3}$ Endoscopy Division, National Cancer Center Hospital, Tokyo,

${ }^{4}$ Takahiro Fujii Clinic, Tokyo, ${ }^{5}$ Hattori GI Endoscopy and

Gastroenterology Clinic, Kumamoto, ${ }^{6}$ Oda GI Endoscopy

and Gastroenterology Clinic, Kumamoto, 'Digestive Disease

Center, Showa University Northern Yokohama Hospital,

Yokohama, ${ }^{8}$ Department of Gastroenterology, Kitasato

University East Hospital, Sagamihara, ${ }^{9}$ Department of

Gastrointestinal Oncology, Osaka Medical Center for

Cancer and Cardiovascular Diseases, Osaka, ${ }^{\circ}$ Division of

Gastroenterology, Department of Medicine, Showa University

School of Medicine, Tokyo, "Department of Gastroenterology.

Saku Central Hospital, Saku, '2Division of Endoscopy, Shizuoka

Cancer Center, Mishima, ${ }^{13}$ Department of Diagnostic

Imaging, Tochigi Cancer Center, Utsunomiya, I'Department

of Molecular-Targeting Cancer Prevention, Graduate

of Molecular-Targeting Cancer Prevention, Graduate

School $M e d$ cal Science, Kyoto Prefectural University of

Medicine, Kyoto, 'Department of Medical Statistics, Shiga

University or Medical Science, Otsu, Pathology and Clinical

${ }^{17 D}$ Department of Surgical and Molecular Pathology, Dokkyo

Medical University School of Medicine, Mibu, ${ }^{18}$ Division

Medical University School or Medicine, Mibu, "Division

of Molecular and Diagnostic Pathology, Niigata University

Graduate School of Medical and Dental Sciences, Niigat,

${ }^{19}$ Aomori Prefectural Hospital, Aomori, Japan

Correspondence: Yasushi Sano

Gastrointestinal Center and Institution of Minimally

Invasive Endoscopic Care (iMEC), Sano Hospital,

2-5-I Shimizugaoka, Tarumi-ku, Kobe,

Hyogo 655-003I, Japan

Tel +81787851000

Fax +81787850077

Email ys_endoscopy@hotmail.com
Background: The Japan Polyp Study (JPS) Workgroup was established in 2000 to evaluate colonoscopic follow-up surveillance strategies. The JPS was a multicenter randomized controlled trial designed to evaluate follow-up surveillance strategies in patients who had undergone two complete colonoscopies for control of colorectal cancer, with removal of all detected polyps. The aim of the present analysis was to assess the patient recruitment and whether the clinical characteristics were adequate for enrollment at the participating centers.

Materials and methods: Among referrals for colonoscopy at the eleven participating centers, all patients who were 40-69 years old, without a family or personal history of familial polyposis, Lynch syndrome, inflammatory bowel disease, or a personal history of polypectomy with unknown histology, and had no invasive colorectal cancer or colectomy, were considered for inclusion from February 2003.

Results: Among 4,752 referrals, a total of 3,926 patients with a mean age of 57.3 (range 40-69) years, including 2,440 (62\%) males, were included in the JPS. The participation rate was $83 \%$. Among them, a total of 2,757 patients who had undergone two complete colonoscopies with removal of all detected polyps were eligible, giving an eligibility rate of $70 \%(2,757$ of 3,926$)$. Among the eligible patients, 2,166 were assigned to randomized groups, and 591 patients to a nonrandomized group. The last steps of data lock, analysis, and complete histopathological assessment based on a pathology review are ongoing.

Conclusion: Eligible patients recruited for the JPS were successfully assigned on the basis of the expected sample-size calculation.

Keywords: colonoscopy, follow-up surveillance strategies, Japan Polyp Study (JPS), study design, multicenter randomized controlled trial

\section{Introduction}

Colorectal cancer is the third-most important cause of cancer mortality in Japan. ${ }^{1}$ Identification and removal of adenomatous polyps and postpolypectomy surveillance are considered to be important for control of colorectal cancer. ${ }^{2,3}$ However, there have been no established recommendations for postpolypectomy colonoscopic surveillance in Japan. In current practice, the intervals between colonoscopies after polypectomy are variable, often being up to a year, and not based on data from randomized clinical trials.

The evolution of colorectal cancer from its precursor lesion, adenoma, was first reported by Morson as the adenoma-carcinoma sequence. ${ }^{4}$ The introduction of colonoscopy provided an opportunity for clarifying this sequence, because of its ability to examine the entire colon and remove polyps for pathological examination. The epidemiology and natural history of adenomas are not only important for choosing 
the optimal follow-up policy after polypectomy but also for evaluating endoscopic screening for colorectal adenomas and cancer. On the other hand, depressed lesions, including some with advanced histology, have been demonstrated in a number of recent series from several Western countries and Japan. ${ }^{5-9}$ However, the clinical significance of depressed lesions in the evolution of colorectal cancer, ie, the so-called de novo pathway, is still controversial. ${ }^{10}$

In the US, the National Polyp Study (NPS), which has been ongoing since 1980, has recommended an interval of at least 3 years between colonoscopic removal of newly diagnosed adenomatous polyps and follow-up examination. . $^{2,3,11}$ Although long-term evaluation of colonoscopic polypectomy in the NPS demonstrated a 53\% reduction in mortality due to colorectal cancer, the study was conducted prior to recent epidemiologic studies documenting the importance of nonpolypoid lesions. Therefore, such techniques as chromoendoscopy, required for diagnosis of nonpolypoid lesions, were not used in the NPS, and this may at least partly explain the discrepancy between the results of the NPS and subsequent epidemiological studies. ${ }^{12,13}$

The Japan Polyp Study (JPS) Workgroup was established in 2000 to evaluate follow-up colonoscopic surveillance strategies, partly supported by a Grant for Scientific Research Expenses for Health and Welfare Programs. ${ }^{14}$ The JPS was a multicenter randomized controlled trial designed to evaluate follow-up surveillance strategies in patients who have undergone two complete colonoscopies for control of colorectal cancer, with the removal of all detected polyps by high-resolution chromoendoscopy, including nonpolypoid lesions. From 2000 to 2002, we confirmed whether randomization was feasible after one complete colonoscopy as far as the cecum, with removal of all detected adenomatous polyps, as was the case in the NPS. Initially, we had investigated a retrospective cohort study to estimate the incidence of advanced neoplasia after initial colonoscopy at six institutes of the JPS Workgroup. ${ }^{14,15}$ Unexpectedly, the study showed higher cumulative incidences of index lesions, which were defined as large adenomatous polyps measuring $>10 \mathrm{~mm}$, adenomas with high-grade dysplasia, or invasive cancer (1 year after initial colonoscopy, 3.2\%, including seven invasive cancers; 3 years after initial colonoscopy, $7.5 \%$, including 13 invasive cancers). ${ }^{16}$ Based on these results, the JPS Workgroup considered it necessary to perform two complete colonoscopies before randomization in order to avoid overlooking any clinically significant lesions. All of the institutional review boards finally agreed that randomization into two groups undergoing either 1) follow-up examination at 1 year and 3 years or 2) follow-up examination at 3 years after two complete colonoscopies as far as the cecum, with removal of all adenomatous polyps detected, was acceptable (Figure 1). ${ }^{16}$

The primary end point of the JPS was to address the hypothesis that after two complete colonoscopies with the removal of all detected polyps, the incidence of index lesions would not be different between patients undergoing two further follow-up examinations and those undergoing just one. The aim of the present analysis was to assess the patient recruitment of the JPS and to determine whether the clinical characteristics were adequate for enrollment at the participating centers.

\section{Materials and methods Organization}

This multicenter prospective study was initiated by the JPS Workgroup, which was established in 2000 in Japan. Eleven participating centers accrued patients for the JPS (Table 1). All entries and follow-up examinations were the responsibility of the study investigators. Quality-assurance programs were instituted in each of the disciplines encompassed by the study. An independent pathology-review team was established at the National Cancer Center (Tokyo, Japan) to examine all resected polyps prospectively, and for each polyp a consensus among the three pathology reviewers (TS, TF, and YA) was reached. The pathologic classification adopted for the JPS was based on the World Health Organization classification. ${ }^{17}$ All depressed lesions and index lesions were reviewed independently by the endoscopic review team at the National Cancer Center. The endoscopic classification was based on the Japanese Society for Cancer of the Colon and Rectum system. ${ }^{18}$ All data obtained on the basis of the JPS protocol were overseen by Medical Support Research (Osaka, Japan).

\section{Sample-size calculation}

For determining the sample size required for a noninferiority trial, we set the incidence of index lesions at $3 \%$ at 3 years after initial examination of a clean colon. We also set the noninferiority margin at $2 \%$ (considered as clinically relevant), the one-sided alpha at $2.5 \%$ (one-sided), and the statistical power at $80 \%$. For each group, 1,142 patients were required, and a total of 2,284 patients were required for the trial. Considering the patient dropout rate (20\%) and patients who did not have polyps (who were not eligible for the randomized trial: $20 \%$ ), we planned to recruit at least 4,000 patients for the study. 


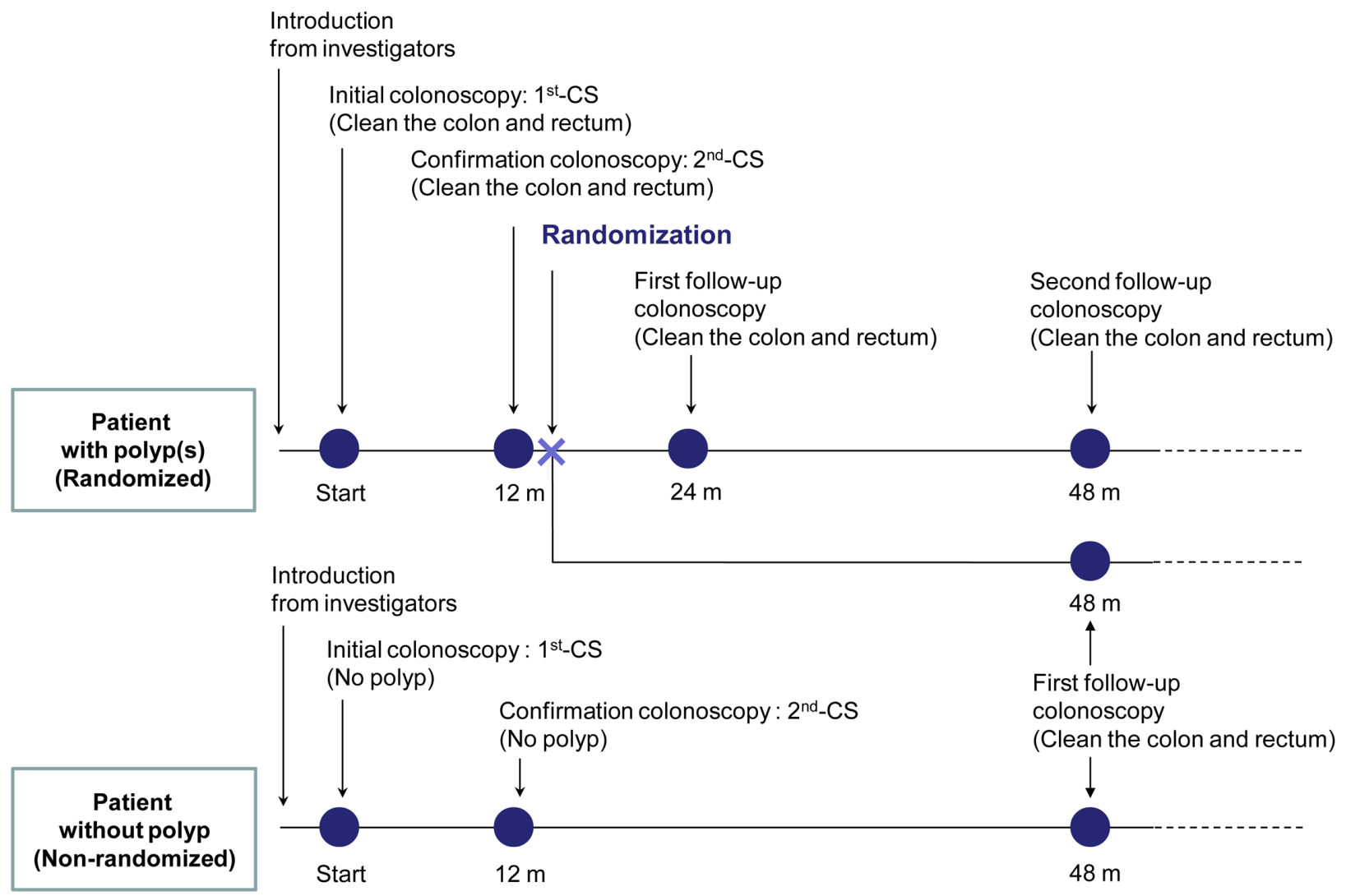

Figure I Design of the Japan Polyp Study.

Abbreviations: $\mathrm{CS}$, colonoscopy; m, months.

Table I Participating, review, and data centers in the Japan Polyp Study

\begin{tabular}{ll}
\hline & $\begin{array}{l}\text { Location } \\
\text { (prefecture) }\end{array}$ \\
\hline Participating center & \\
I. National Cancer Center Hospital & Tokyo \\
2. National Cancer Center Hospital East & Chiba \\
3. Saku Central Hospital & Nagano \\
4. Showa University Hospital & Tokyo \\
5. Showa University Northern Yokohama Hospital & Kanagawa \\
6. Kitasato University East Hospital & Kanagawa \\
7. Shizuoka Cancer Center & Shizuoka \\
8. Hattori Gl Endoscopy and Gastroenterology Clinic & Kumamoto \\
9. Osaka Medical Center for Cancer & Osaka \\
and Cardiovascular Diseases & \\
I0. Takahiro Fujii Clinic & Tokyo \\
II. Tochigi Cancer Center* & Tochigi \\
Pathology review & \\
I. National Cancer Center Hospital & Tokyo \\
2. Dokkyo Medical University & Tochigi \\
3. Niigata Medical University & Niigata \\
Endoscopic review & \\
I. National Cancer Center Hospital & Tokyo \\
Data Center & \\
I. Medical Research Support & Osaka \\
\hline
\end{tabular}

Note: *Since 2005

\section{Informed consent}

Information on the protocol was given to all patients, and informed consent was secured after the respective interventions. In regular checkups, there was no discrimination according to agreement/refusal to take part in the study, or any drawback resulting from withdrawal. Only patients who gave informed consent were included in the study.

\section{Patient accrual}

All patients referred for colonoscopy at the eleven participating centers shown in Table 1, who were 40-69 years old, without a family or personal history of familial polyposis, Lynch syndrome, or inflammatory bowel disease, or a personal history of polypectomy with unknown histology, and without invasive colorectal cancer or colectomy, were considered for inclusion from February 2003.

Patients were excluded if colonoscopy revealed colorectal cancer invading beyond the muscularis mucosae, or a sessile adenoma with a base exceeding $3 \mathrm{~cm}$ in diameter. The data collected for these patients consisted of the reason for referral, the outcome of the examination, and certain items of 
demographic information. Patients were eligible if they had undergone two complete colonoscopies (first CS and second CS) as far as the cecum, with removal of all polyps detected. The patients were assigned to two groups: 1) a nonrandomized group (patients without polyps), and 2) a randomized group (patients with polyps). Data for all patients in these two groups included detailed demographics, medical history, and information on the procedure employed and the individual polyp(s) resected. Nonrandomized patients included those who had no neoplastic lesions were enrolled at 3 years only after undergoing two complete colonoscopies (first CS and second CS).

All randomized patients were randomly assigned to undergo follow-up colonoscopy at 1 and 3 years, or at 3 years only. For randomization, we used a dynamic allocation scheme to ensure balance between the two groups over time. ${ }^{19}$ There were five prognostic factors: 1) institution, 2) $\operatorname{sex}$ (male/female), 3) age category ( $\geq 65$ years and $<65$ years), 4) risk stage of colon cancer - a) patients with polyps measuring $\leq 5 \mathrm{~mm}$ only on two complete colonoscopies (first CS and second CS), b) patients with polyps measuring $\geq 6 \mathrm{~mm}$ on two complete colonoscopies, independently of the presence of polyps measuring $\leq 5 \mathrm{~mm}$, and c) patients with any intramucosal cancer, independently of the presence and size of the polyp on colonoscopy - and 5) history of colonoscopy, chosen for balance between the groups. The study design of the JPS is shown in Figure 1.

\section{Follow-up}

All patients were contacted by post 2 months before their scheduled follow-up month, with a request to undergo the follow-up examination.

\section{Statistical analysis}

Basic descriptive analyses of the pool of patients referred for colonoscopy and those eligible for the surveillance study are presented. The percentages were recorded in the categorical data (eg, sex and reason for referral) for each center. Means and standard deviation were used for quantitative data (eg, age).

\section{Ethical considerations}

The protocol was reviewed a priori and approved by the institutional review boards at all of the participating hospitals in 2003. This trial is registered, and details are available at http://www.umin.ac.jp (UMIN C000000058), where the trial protocol can be accessed.

\section{Funding}

The study was supported by Grants-in-Aid for Clinical Cancer Research (13S-8, 16S-33, 20S-12, and 23S-8) from the Ministry of Health, Labour and Welfare, Japan. The JPS website is http://www.jps21.jp/index.html (Japanese only).

\section{Results}

A flowchart for the JPS is shown in Figure 2.

\section{Patient recruitment for the JPS}

Patient accrual began in February 2003 and ended in December 2006. A total of 4,752 patients were referred to the eleven participating centers for colonoscopy, and none of them had a history of familial polyposis, Lynch syndrome, or inflammatory bowel disease, a personal history of polypectomy with unknown histology, or had had invasive colorectal cancer or colectomy. A total of 3,926 patients with a mean age of 57.3 (range 40-69) years, including 2,440 (62\%) males, who consented to join the study were included in the JPS (Table 2). The participation rate was $83 \%(3,926$ of 4,752$)$.

\section{Reason for referral}

Although most patients were referred for a number of reasons, each patient was categorized according to the main finding on which the referral was based (Table 3 ). The predominant reason for referral was a positive result for fecal occult blood testing (FOBT; 37.2\%), followed by overt symptoms $(16.9 \%)$.

\section{First colonoscopy}

A total of 3,895 patients underwent colonoscopy, among whom 178 were excluded due to 1 ) invasive cancers (109 patients: 45 with advanced cancer, 66 with submucosal invasive cancer, and three with intramucosal cancer, with a certain degree of overlap), 2) lesions more than $30 \mathrm{~mm}$ in diameter, or for which surgery was indicated (16 patients: nine with adenoma, seven with intramucosal cancer), 3) carcinoid tumor (seven patients), and 4) other reasons (46 patients: inflammatory bowel disease/poor bowel preparation/failure to clean the colon or total colonoscopy). A total of 930 patients dropped out before receiving the second CS in spite of postal notification. The prevalence of patients with invasive colorectal cancer was $2.8 \%$ (109 of 3,895 ).

\section{Second colonoscopy}

A total of 2,787 patients underwent colonoscopy at 1 year after the first CS. A total of 30 patients were excluded due to 1) invasive cancer (four patients: one with advanced cancer, 


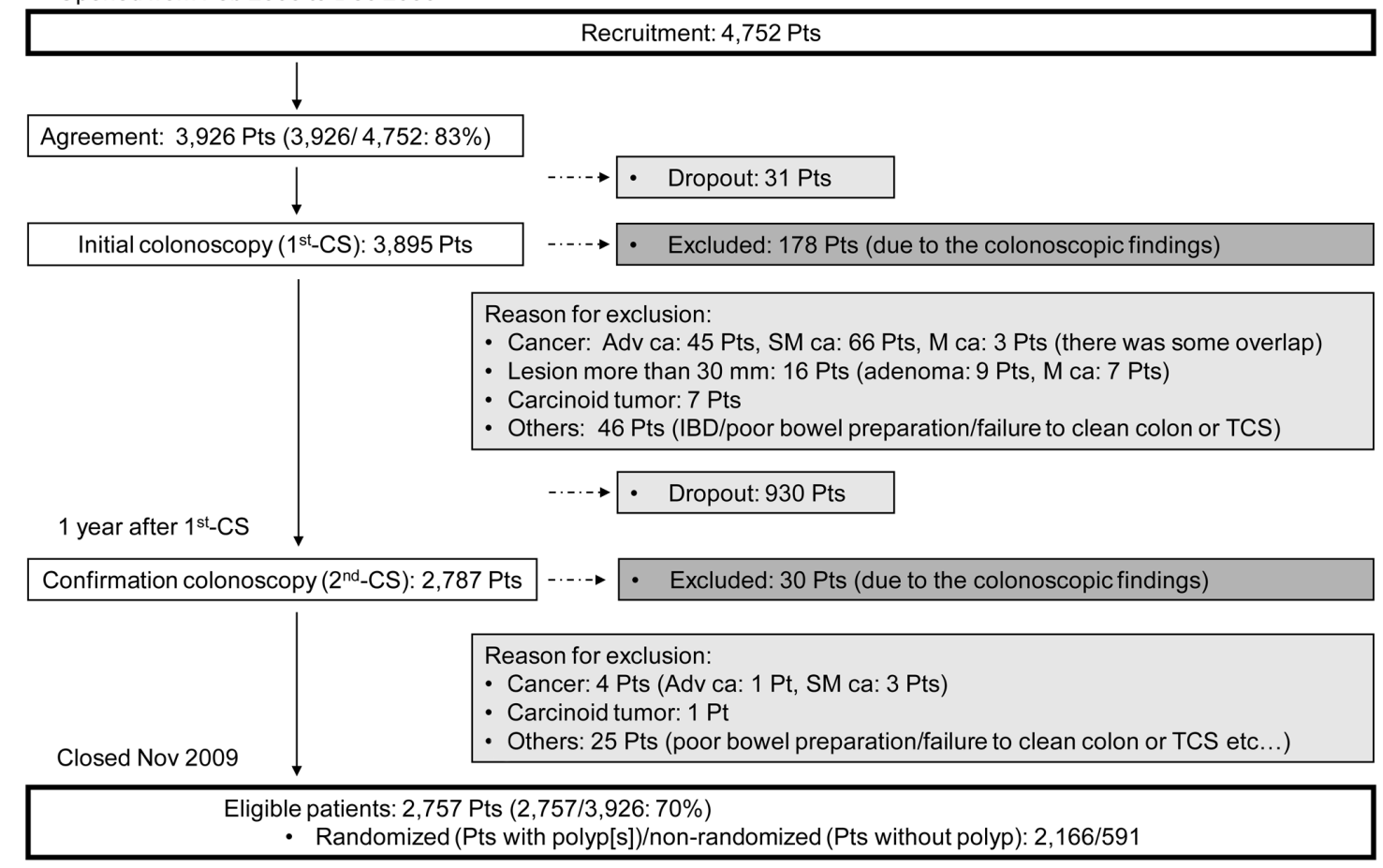

Figure 2 Japan Polyp Study flow.

Abbreviations: Pts, patients; CS, colonoscopy; Adv, advanced; ca, cancer; SM, submucosal invasive; M, intamucosal; IBD, inflammatory bowel disease; TCS, total colonoscopy.

three with submucosal invasive cancer), 2) carcinoid tumor (one patient), and 3 ) other reasons (25 patients: poor bowel preparation/failure to clean the colon or total colonoscopy). The prevalence of patients with interval invasive colorectal cancer was $0.14 \%$ (four of 2,787 ).

\section{Patients eligible for the JPS}

A total of 2,757 patients who underwent two complete colonoscopies (first CS and second CS) as far as the cecum, with removal of all polyps detected, were eligible. The eligibility rate was $70 \%(2,757$ of 3,926$)$. Among the eligible patients, 2,166 were assigned to randomized patient groups (patients with polyp[s]) and 591 patients to a nonrandomized patient group (patients without polyp).

\section{Discussion}

The NPS, which was started in the USA in 1980, showed that the removal of all polyps by colonoscopy reduces the incidence of colorectal cancer, and recommended an interval of at least 3 years between colonoscopic removal of newly diagnosed adenomatous polyps and subsequent follow-up examination. ${ }^{2,3}$ In Japan, however, there are no established recommendations for postpolypectomy colonoscopic surveillance based on reliable evidence.
The JPS Workgroup was established in 2000 to evaluate colonoscopic follow-up surveillance strategies under a Grant for Scientific Research Expenses for Health and Welfare Programs. The JPS is the largest clinical study conducted in ethnic Japanese communities, documenting for the first time follow-up surveillance strategies for patients who have undergone two complete colonoscopies for control of colorectal cancer, with the removal of all detected polyps. ${ }^{16}$ It will provide preexisting comorbidity data, including the prevalence of both flat and depressed colorectal lesions, the quality of colonoscopy, and the risk of colon cancer. Furthermore, these follow-up data will help to clarify the long-term impact of colonoscopic removal on mortality due to colorectal cancer. The evidence will form a basis for the surveillance and management of colorectal cancer in community-dwelling individuals who undergo colonoscopy in Japan.

Although the subjects in the JPS underwent two complete colonoscopies before randomization, the participation rate was high (83\%). However, 24.5\% (961 of 3,926) of the patients dropped out from the study before randomization, mostly after the first CS. One possible reason for the high dropout rate was that all examinations were performed under the national health insurance scheme. Patients had to pay expenses ranging from US\$35 to $\$ 80$ per colonoscopy, or $\$ 100-\$ 300$ 


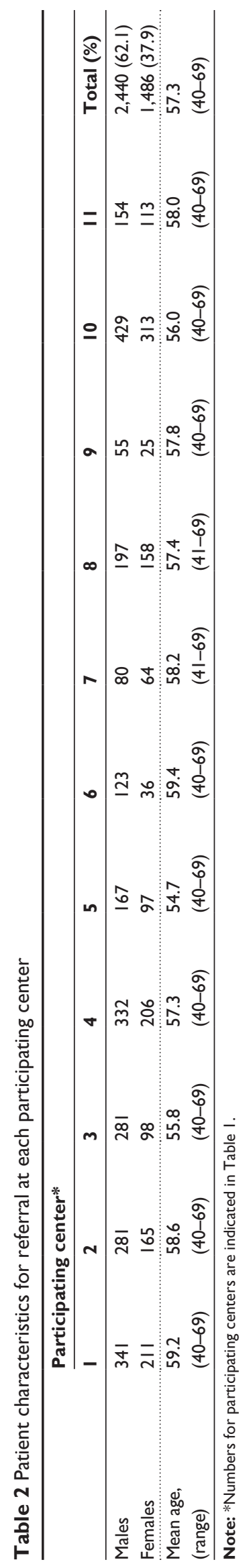

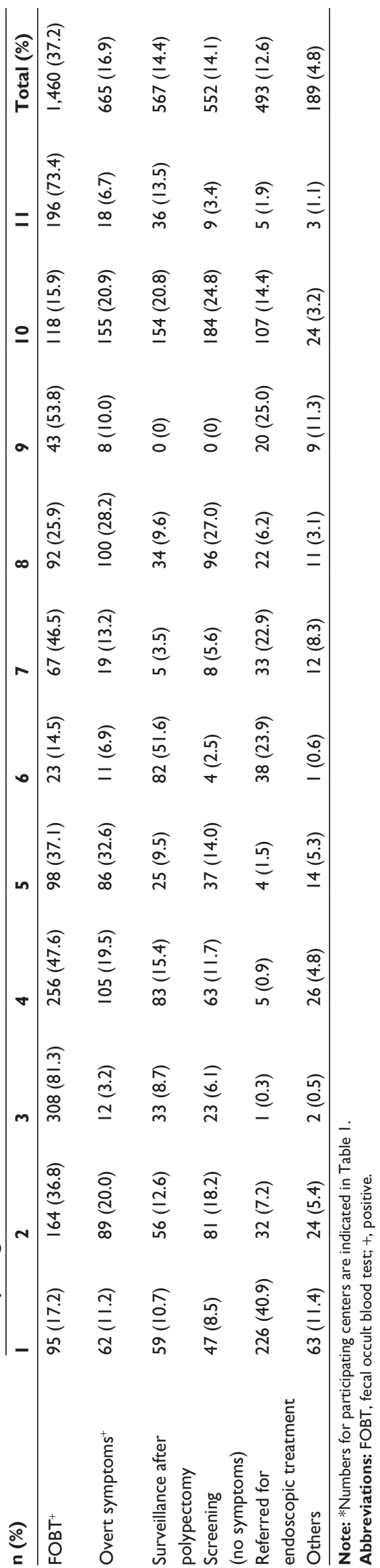


for polypectomy. However, we had to consider whether two colonoscopies are realistically tolerable for Japanese patients after completing the JPS. Finally, a total of 2,166 patients were assigned to randomized patient groups, and these eligible patients recruited for the JPS were successfully assigned in line with the expected sample-size calculation.

The sex distribution of the JPS subjects, with a mean age of 57.3 years, showed a male predominance at all participating centers. A recent study reported that male sex was associated with an increase in adenoma detection, with a statistically significant odds ratio of 1.89 (95\% confidence 1.78-1.99) compared to females. ${ }^{20}$ The first reason for the male predominance was that most of the patients had some risk factors with one or more adenomas for referral $\left(\mathrm{FOBT}^{+}\right.$, $37.2 \%$; surveillance after polypectomy, $14.4 \%$; referred for endoscopic treatment, $12.6 \%$ ). The second reason was that the sex distribution of patients mentioned earlier accidentally showed a male predominance. The incidence of male sex was $63 \%$ in $\mathrm{FOBT}^{+}, 67 \%$ in surveillance after polypectomy, and $72 \%$ in referred for endoscopic treatment, respectively. However, the sex distribution and mean age of the patients (male/female/age: $59.1 \% / 40.9 \% / 60$ years) recruited for the NPS were almost the same as for the JPS. ${ }^{11}$ The final results of the JPS should be compared with the outcome of the NPS to assess the effectiveness of two colonoscopies with removal of all polyps before randomization for reduction of the incidence of advanced neoplasia.

This study had some limitations. We often used highmagnification endoscopes, because these are commonly available at Japanese academic centers. ${ }^{5,6}$ However, there was no disease entity of sessile serrated adenoma/polyps (SSA/P) at the beginning of the JPS, and we did not evaluate the criteria for distinguishing SSA/P from hyperplastic polyps, because no pathologic gold standard for diagnosis has yet been fully established. ${ }^{21}$ Recently, serrated lesions of the colorectum were considered as the precursors of perhaps a third of colorectal cancers. Cancers arising in serrated lesions are usually in the proximal colon, and account for a disproportionate fraction of cancer identified after colonoscopy. Rex et al with an expert panel recommended complete removal of all serrated lesions, except for diminutive sigmoid or rectal lesions. ${ }^{22}$ Interval cancers arising in serrated lesions may not be prevented completely in the JPS, because serrated lesions, including SSA/P, were not always removed.

\section{Conclusion}

All patients recruited for the JPS were successfully assigned in line with the expected sample-size calculation. The last steps of data calculation/statistical processing at the data center and complete histopathological assessment by pathology review are ongoing. It is anticipated that the data will help to establish not only effective surveillance strategies after removal of all detected polyps but also reveal details of preexisting comorbidity, including prevalence of colorectal lesions, quality of colonoscopy, and the risk of colon cancer. Surveillance continues to evaluate the long-term effect of colonoscopic polypectomy in the JPS on mortality from colorectal cancer.

\section{Acknowledgments}

We thank all investigators who cooperated in recruiting patients at the eleven participating centers, Dr Tetsuichiro Muto (Cancer Institute Hospital of the JFCR, Tokyo, Japan), and Dr Hiroshi Saito (Research Center for Cancer Prevention and Screening, National Cancer Center, Tokyo, Japan), for his valuable initial advice regarding the organization of the Japan Polyp Study Workgroup.

\section{Disclosure}

The authors report no conflicts of interest in this work.

\section{References}

1. Saito H. Screening for colorectal cancer: current status in Japan. Dis Colon Rectum. 2000;43:S78-S84.

2. Winawer SJ, Zauber AG, Ho MN, et al. Prevention of colorectal cancer by colonoscopic polypectomy. The National Polyp Study Workgroup. N Engl J Med. 1993;329:1977-1981.

3. Winawer SJ, Zauber AG, O'Brien MJ, et al. Randomized comparison of surveillance intervals after colonoscopic removal of newly diagnosed adenomatous polyps. The National Polyp Study Workgroup. $N$ Engl $J$ Med. 1993;328:901-906.

4. Morson BC. President's address. The polyp-cancer sequence in the large bowel. Proc R Soc Med. 1974;67:451-457.

5. Kudo S. Endoscopic mucosal resection of flat and depressed type of early colorectal cancer. Endoscopy. 1993;25:455-461.

6. Fujii T, Rembacken BJ, Dixon MF, Yoshida S, Axon AT. Flat adenomas in the United Kingdom: are treatable cancers being missed? Endoscopy. 1998;30:437-443.

7. Rembacken BJ, Fujii T, Cairns A, et al. Flat and depressed colonic neoplasms: a prospective study of 1000 colonoscopies in the UK. Lancet. 2000;355:1211-1214.

8. Jaramillo E, Watanabe M, Slezak P, Rubio C. Flat neoplastic lesions of the colon and rectum detected by high-resolution video endoscopy and chromoendoscopy. Gastrointest Endosc. 1995;42:114-122.

9. Tsuda S, Veress B, Tóth E, Fork FT. Flat and depressed colorectal tumors in a southern Swedish population: a prospective chromoendoscopic and histopathological study. Gut. 2002;51:550-555.

10. Soetikno RM, Kaltenbach T, Rouse RV, et al. Prevalence of nonpolypoid (flat and depressed) colorectal neoplasms in asymptomatic and symptomatic adults. JAMA. 2008;299:1027-1035.

11. Winawer SJ, Zauber AG, O'Brien MJ, et al. The National Polyp Study. Design, methods, and characteristics of patients with newly diagnosed polyps. The National Polyp Study Workgroup. Cancer. 1992;70: $1236-1245$.

12. Zauber AG, O’Brien MJ, Winawer SJ. On finding flat adenomas: is the search worth the gain? Gastroenterology. 2002;122:839-840. 
13. O'Brien MJ, Winawer SJ, Zauber AG, et al. Blinded assessment of the flat adenoma in the National Polyp Study (NPS) does not demonstrate an excess risk for high grade dysplasia initially or for advanced adenomas at surveillance. Gastroenterology. 2001;120:A96.

14. Fujii T, Sano Y, Iishi H, et al. Colorectal cancer screening in Japan: results of the multicenter retrospective cohort study. Gastroenterology. 2002;122:A481.

15. Matsuda T, Fujii T, Sano Y, et al. Five-year incidence of advanced neoplasia after initial colonoscopy in Japan: a multicenter retrospective cohort study. Jpn J Clin Oncol. 2009;39:435-442.

16. Sano Y, Fujii T, Oda Y, et al. A multicenter randomized controlled trial designed to evaluate follow-up surveillance strategies for colorectal cancer: the Japan Polyp Study. Dig Endosc. 2004;16:376-378.

17. Hamilton SR, Aaltonen LA, editors. WHO Classification of Tumors. Tumors of the Digestive System. Lyon: IARC; 2000.
18. Japanese Society for Cancer of the Colon and Rectum. General Rules for Clinical and Pathological Studies on the Colon, Rectum and Anus. 6th ed. Tokyo: Kanehara; 1998.

19. Green S, Beneditti J, Crowley J. Clinical Trials in Oncology. Boca Raton (FL): Chapman and Hall/CRC; 1997.

20. Lee TJ, Rees CJ, Blanks RG, et al. Colonoscopic factors associated with adenoma detection in a national colorectal cancer screening program. Endoscopy. 2014;46:203-211.

21. Torlakovic E, Skovlund E, Snover DC, Torlakovic G, Nesland JM. Morphologic reappraisal of serrated colorectal polyps. Am J Surg Pathol. 2003;27:65-81.

22. Rex DK, Ahnen DJ, Baron JA, et al. Serrated lesions of the colorectum: review and recommendations from an expert panel. Am J Gastroenterol. 2012;107(9):1315-1329.

\section{Publish your work in this journal}

The Open Access Journal of Clinical Trials is an international, peerreviewed, open access journal publishing original research, reports, editorials, reviews and commentaries on all aspects of clinical trial design, management, legal, ethical and regulatory issues, case record form design, data collection, quality assurance and data auditing methodologies. The manuscript management system is completely online and includes a very quick and fair peer-review system, which is all easy to use. Visit http://www.dovepress.com/testimonials.php to read real quotes from published authors.

Submit your manuscript here: http://www.dovepress.com/open-access-journal-of-clinical-trials-journal 